phys. stat. sol. (b) 231, No. 1, 132-148 (2002)

\title{
On the Theory of X-Ray Diffraction and X-Ray Standing Waves in the Multilayered Crystal Systems
}

\author{
V. G. $\mathrm{KoHN}^{1}$ ) \\ Russian Research Centre "Kurchatov Institute”, 123182 Moscow, Russia
}

(Received February 27, 2001; in revised form October 23, 2001; accepted October 25, 2001)

Subject classification: 61.10.Dp; 78.70.Ck; S7.11

\begin{abstract}
The theory for the Bragg dynamical X-ray diffraction and the yield of the secondary radiation scattered via incoherent channels under conditions of X-ray diffraction (X-ray standing wave technique) is developed for the crystal systems composed from many layers. The formulae are derived in a form suitable for a computer simulation of the experimental angular or energy spectra as well as for a determination of unknown parameters via fitting. The Bragg case (reflection) and the Laue case (transmission) are considered within the same approach. The universal computer program is created and its operation is demonstrated on two examples. It is shown that the model of the multilayered crystal system may be a useful tool for analyzing a lot of problems where the one-dimensional crystal-lattice distortions of different kinds influence the rocking curve and the curve of the secondary radiation yield. In the first example the $\operatorname{In}_{0.5} \mathrm{Ga}_{0.5} \mathrm{P}$ epitaxial film on the GaAs substrate is considered. The experimental angular spectra of the X-ray reflectivity and the yield of the In $\mathrm{L}_{\alpha}$ and $\mathrm{P} \mathrm{K}_{\alpha}$ fluorescence in the Bragg case are described by fitting the parameters of the structure. In the second example the Laue case of X-ray diffraction in a sample having a monotonously increasing (decreasing) shift of the Bragg angle with increasing depth is analyzed. The phenomenon of the complete switching of the X-ray beam from the incident direction to the direction of reflection is calculated and discussed.
\end{abstract}

\section{Introduction}

The technique of X-ray standing waves (XSW) consists of measuring the yield of the secondary radiation scattered incoherently under conditions of the two-beam dynamical diffraction of X-rays in a near-perfect crystal. The first studies were done about thirty years ago. Many different channels of incoherent scattering were studied. We would like to point out the first studies of fluorescence [1, 2] and photoelectron emission $[3,4]$. For these channels, the secondary radiation arises as a result of the photoelectric absorption of X-ray quanta by individual atoms of a crystal. The atoms perceive the X-ray standing wave as a coherent superposition of the transmitted and the reflected plane waves. That is why the secondary radiation yield (SRY) changes drastically within the Bragg diffraction region in the angular spectra (the rocking curves) or the energy spectra. The specific shape of the curves depends on the probability for the secondary radiation emerged by an atom at some depth inside the crystal to reach the crystal surface.

The probability function for fluorescent photons to reach the surface from the definite depth $z$ is equal to $\exp \left(-\mu_{\mathrm{yi}} z\right)$. The characteristic depth $L_{\mathrm{yi}}$ of the fluorescent radiation is well known as $\mu_{\mathrm{yi}}^{-1}$ where $\mu_{\mathrm{yi}}$ is a linear absorption coefficient. Typically, the value $L_{\mathrm{yi}}$ is longer than the extinction length $L_{\mathrm{ex}}$. The latter is determined as a depth

1) Corresponding author; e-mail: kohn@kurm.polyn.kiae.su 
of decaying the X-ray standing wave inward the crystal at the middle of the Bragg peak of the rocking curve in the Bragg case. As a result, the angular dependence of the $\mathrm{X}$-ray penetration depth inward the crystal masks the angular dependence of the intensity of the X-ray standing wave at the atoms which are the sources of SRY. The probability function for electrons escaped due to photoelectric absorption has a more complicated form. It is studied in detail in [5] by means of the Monte-Carlo computer simulation. In Ref. [6] this function is proposed to have approximately an exponential form, at least, for the measurements integral over the electron energy. However, the value $\mu_{\mathrm{yi}}$ has now a more complicated physical meaning. As a rule, it applies $L_{\mathrm{yi}} \ll L_{\mathrm{ex}}$ in the case of photoelectron emission. Therefore, the experimental curves show just the intensity of the X-ray standing wave at the atom position. In the case of fluorescence the same situation arises if a signal from the impurity atoms located near the surface of a sample is recorded [2].

It was discovered later [7-9] that the XSW technique is very sensitive to the structure of a subsurface layer disturbed due to some external perturbation, e.g. ion implantation, epitaxial film growth etc. if the condition $L_{\mathrm{yi}} \ll L_{\mathrm{ex}}$ is fulfilled. The theoretical explanation of this phenomenon is done in [10]. See [11, 12] for more detailed information. For years the X-ray standing wave technique has become a routine tool for studying impurity or basic atom location in the subsurface layer of the near-perfect crystal (see, e.g. [13-17] and references therein). Both X-ray tube generators and synchrotron radiation beamlines are used in experiments. In addition to the nondispersive $(n,-n)$ configuration of the two-crystal scheme the dispersive XSW geometries are used [17, 18]. The Laue case is also investigated [19].

The results of many experimental studies of the angular dependence of the secondary radiation yield $Y(\Delta \theta)$ are discussed in terms of the XSW intensity at a mean atom position with respect to the XSW nodes called the coherent position $P_{\mathrm{c}}$. A deviation of the real position of an individual atom from the mean position is taken into account through the parameter called coherent fraction $f_{\mathrm{c}}$. Thus, the experimental curves are fitted by a simple formula [20]

$$
Y(\Delta \theta)=Z(\Delta \theta)\left\{1+R(\Delta \theta)+2 f_{\mathrm{c}} P \sqrt{R(\Delta \theta)} \cos \left[v(\Delta \theta)-\Theta_{\mathrm{c}}\right]\right\}
$$

where $Z(\Delta \theta)$ is a normalized effective thickness of SRY, $R(\Delta \theta)$ is a reflectivity, $P$ is a polarization constant, $v(\Delta \theta)$ is the XSW phase and $\Theta_{\mathrm{c}}=2 \pi P_{\mathrm{c}}$. Formula (1) is rather useful in some simple cases of the photoelectron yield with a very short escape depth $L_{\mathrm{yi}}$. On the other hand, it cannot be used for the cases when the thickness of a disturbed layer is comparable with $L_{\mathrm{yi}}$ or $L_{\mathrm{ex}}$.

In the general case, as was indicated for the first time in $[10,21]$, the solution of the Takagi equations must be used for calculating the local electric field of X-rays inside the crystal with a disturbed subsurface layer. In addition, one has to take into account the probability function $P_{\mathrm{yi}}(z)$ for the secondary radiation in order to reach the surface of the sample from the depth $z$. Of course, one has to integrate the SRY over the depth of the sample. The Takagi equations for the sample with variable structure parameters of the general profile can be solved only numerically. The way of solving of the problem is proposed in [21]. However, very often the sample consists of several layers having approximately constant structure parameters with sharp interfaces between them. This is valid for one epitaxial film on a substrate [9] or for the isotopically different layer on a substrate of normal isotopic composition (see [16, 17] and references 
therein). For these cases, the model of the multilayered crystal system is useful. Such model allows to use the recurrent relations for different layers numerically, whereas the Takagi equations for the individual layers having the constant structure parameters are solved analytically. On the other hand, even the general case may be considered if a sample is approximated by a crystal system consisting of many layers of different thicknesses.

Such approach was considered in [6] for the Bragg case and in [19] for the Laue case as a tool for analyzing specific experimental results. Here we present the general formulae for calculating the XSW spectra and X-ray reflectivity in the case of the multilayered crystal system. The formulae may be used as a basis for a computer program. The code of our program is written in FORTRAN. Therefore, it may be used in the operating systems DOS (Windows) and UNIX. We demonstrate possibilities of the approach on two particular examples. In the first example the Bragg case of X-ray diffraction in the $\mathrm{In}_{0.5} \mathrm{Ga}_{0.5} \mathrm{P}$ epitaxial film on the GaAs substrate is analyzed more accurately compared to Ref. [22]. In the second example the Laue case of X-ray diffraction in the sample with the monotonously increasing (decreasing) parameter of a deviation from the Bragg condition is considered. The phenomenon of the complete switching of the X-ray beam from the incident direction to the direction of reflection is calculated and discussed.

\section{Solution for One Crystal Layer}

We consider a sample having a lamina-like shape. We use the Cartesian coordinate system with the $z$-axis directed along the internal normal to the entrance surface. We analyze the two-beam case of X-ray diffraction in which the Bragg condition is met only for one reciprocal lattice vector $\mathbf{h}$. Therefore, we try the solution of the Maxwell's equation in the form

$$
\mathbf{E}(\mathbf{r}, \omega)=\exp \left(i \mathbf{k}_{0} \mathbf{r}\right)\left[\mathbf{e}_{0} E_{0}(z)+\mathbf{e}_{h} E_{h}(z) \exp (i \mathbf{h r})\right]
$$

where $\mathbf{e}_{0}, \mathbf{e}_{h}$ are the unit vectors of polarization, $\mathbf{k}_{0}$ is the wave-vector of the incident plane wave in the air, $\left|\mathbf{k}_{0}\right|=K$ where $K=\omega / c=2 \pi / \lambda, c$ is the speed of light and $\lambda$ is the wavelength of the X-rays. The complex functions $E_{0, h}(z)$ are slowly varying in the space compared to the exponential $\exp (\boldsymbol{i h r})$. We assume the incident wave to be a plane-polarized wave. This is valid for synchrotron radiation. In the case of nonpolarized radiation one has to consider two standard polarization states and then to average the intensity over the polarizations.

The integration of the Maxwell's equation over the unit cell allows us to write the set of equations for $E_{0}(z)$, and $E_{h}(z)$. As a result, we obtain the Takagi equations in the form [10]

$$
\begin{aligned}
& 2 \gamma_{0} \frac{\mathrm{d} E_{0}}{\mathrm{~d} z}=i K\left\{\chi_{0} E_{0}+C \chi_{\bar{h}} \exp (i \varphi-W) E_{h}\right\}, \\
& \left.2 \gamma_{h} \frac{\mathrm{d} E_{h}}{\mathrm{~d} z}=i K\left\{\chi_{0}-\alpha\right] E_{h}+C \chi_{h} \exp (-i \varphi-W) E_{0}\right\},
\end{aligned}
$$

where $\gamma_{0}=k_{0 z} / K$, and $\gamma_{h}=k_{h z} / K$, are the geometrical parameters, $\alpha=\left[\mathbf{k}_{h}^{2}-\mathbf{k}_{0}^{2}\right] / K^{2}$ is the parameter of deviation from the Bragg condition, $\mathbf{k}_{h}=\mathbf{k}_{0}+\mathbf{h}$ is the wave vector of the diffracted wave, $C=\left(\mathbf{e}_{0} \mathbf{e}_{h}\right)$ is the polarization factor, and $\varphi(z)=\mathbf{h u}(z)$ is an additional phase due to a mean displacement of atoms. The values $\chi_{0}, \chi_{h}$, and $\chi_{\bar{h}}$ are the Fourier coefficients of the crystal susceptibility with the reciprocal lattice vectors 0 , $\mathbf{h}$, 
$-\mathbf{h}$. Finally, factor $\exp [-W(z)]$ describes the dephasing of the scattered wave owing to random displacements of atoms from the mean value at the depth $z$. The factor was introduced for the first time in $[10,23]$ and was called the static Debye-Waller factor on the analogy of the well-known thermal Debye-Waller factor. The latter is incorporated into the crystal susceptibility.

The kind of the boundary conditions for Eq. (3) depends on the sign of the geometrical parameter $\gamma_{h}$. In the Laue case, when $\gamma_{h}>0$, the diffracted beam escapes from the crystal plate through the back surface and is absent at the entrance surface. Therefore, we have $E_{0}(0)=1$ and $E_{h}(0)=0$. We assume the entrance surface to be described by the equation $z=0$ and the incident intensity to be normalized to unity. In the Bragg case, when $\gamma_{h}<0$, the diffracted beam escapes from the crystal through the entrance surface and is absent at the back surface. So we have $E_{0}(0)=1$ and $E_{h}(d)=0$, where $d$ is the thickness of the crystal plate.

\subsection{The local reflection amplitude}

We want to consider the Bragg and Laue cases simultaneously. The boundary conditions for the Bragg case do not allow us to obtain the wave fields from the entrance surface step by step. It is convenient to find a ratio of the functions $E_{h}(z)$ and $E_{0}(z)$ first. We introduce the local reflection amplitude

$$
R(z)=\frac{E_{h}(z)}{E_{0}(z)} \frac{\exp [i \varphi(z)]}{Y \beta^{1 / 2}}
$$

and consider the nonlinear equation from Eqs. (3)

$$
\frac{\mathrm{d} R(z)}{\mathrm{d} z}=-\frac{2 i s}{L_{\mathrm{ex}}}\left[y-y_{\varphi}(z)+i y_{0}\right] R(z)+\frac{i C_{1}}{L_{\mathrm{ex}}}\left[s+R^{2}(z)\right]
$$

where the following quantities are introduced:

$$
\begin{aligned}
& L_{\mathrm{ex}}=\frac{\lambda \gamma_{0}}{\pi \beta^{1 / 2} X^{\prime}}, \quad \beta=\frac{\gamma_{0}}{\left|\gamma_{h}\right|}, \quad X=\left(\chi_{h} \chi_{\bar{h}}\right)^{1 / 2}=X^{\prime}+i X^{\prime \prime}=X^{\prime}(1-i p), \\
& Y=\left(\frac{\chi_{h}}{\chi_{\bar{h}}}\right)^{1 / 2}=|Y| \exp \left(i \Phi_{Y}\right), \quad y=-\frac{\left[\alpha \beta-s \chi_{0}^{\prime}(1+s \beta)\right]}{2 \beta^{1 / 2} X^{\prime}}, \\
& y_{0}=\frac{s \chi_{0}^{\prime \prime}(1+s \beta)}{2 \beta^{1 / 2} X^{\prime}}, \quad y_{\varphi}(z)=s \frac{L_{\mathrm{ex}}}{2} \frac{\mathrm{d} \varphi(z)}{\mathrm{d} z}, \quad C_{1}=C(1-i p) \exp (-W) .
\end{aligned}
$$

Here $\chi_{0}=\chi_{0}^{\prime}+i \chi_{0}^{\prime \prime}$. We use $a^{\prime}$ and $a^{\prime \prime}$ for the real and imaginary parts of the complex value $a$. The parameter $s$ is equal to 1 for the Bragg case and -1 for the Laue case. The boundary conditions for the reflection amplitude are as follows: $R(0)=0$ in the Laue case and $R(d)=0$ in the Bragg case.

There are two ways of changing the parameter of deviation from the Bragg condition. The first one is the variation of the angle of incidence $\Delta \theta$ of the X-ray beam, keeping constant the energy of X-ray photons. In this case we have

$$
y=C_{y \theta} \Delta \theta, \quad y_{\varphi}(z)=C_{y \theta} \Delta \theta_{\mathrm{B}}(z), \quad C_{y \theta}=\pi \frac{L_{\mathrm{ex}}}{\lambda\left|\gamma_{h}\right|} \sin 2 \theta_{\mathrm{B}}
$$


where $\theta_{\mathrm{B}}$ is the Bragg angle in the perfect crystal while $\Delta \theta_{\mathrm{B}}(z)$ is a local shift of the Bragg angle at the depth $z$ due to distortions of the crystal lattice. The angle of rotation $\Delta \theta$ is counted in the direction opposite to the direction of the reciprocal lattice vector h. The second way is a change of the energy of X-ray photons, keeping constant the direction of the beam. In this case

$$
y=C_{y \omega} \Delta \hbar \omega, \quad y_{\varphi}(z)=C_{y \omega} \Delta \hbar \omega_{\mathrm{B}}(z), \quad C_{y \omega}=\frac{L_{\mathrm{ex}}}{\hbar c\left|\gamma_{h}\right|} \sin ^{2} \theta_{\mathrm{B}}
$$

where $\hbar=h / 2 \pi, h$ is the Planck constant, $\hbar \omega_{\mathrm{B}}$ is the Bragg energy of X-ray photons, whereas $\Delta \hbar \omega_{\mathrm{B}}(z)$ is a local shift of the Bragg energy at the depth $z$ due to distortions. The origin of the axis of $y$ corresponds to the centre of the diffraction peak for a perfect crystal. The $\Delta \theta$ dependence is usual for the experiments in the nondispersive twocrystal scheme with an X-ray tube as a source. The $\Delta \hbar \omega$ dependence may be used in the experiments with synchrotron radiation, including the back diffraction with the Bragg angle $\pi / 2$. We note that the parameter $y_{0}$ can also be expressed through the dimensionless variables

$$
y_{0}=\frac{s}{4 \gamma_{0}} \mu_{0} L_{\mathrm{ex}}(1+s \beta)
$$

where $\mu_{0}=2 \pi \chi_{0}^{\prime \prime} / \lambda$ is a linear absorption coefficient.

The approach based on the direct numerical solution of the differential Eq. (5) was proposed in [21] (see also [11]). We note that such an approach has a disadvantage for the crystal samples containing thick layers with approximately the same parameters, if the difference between various layers is large. The point is that Eq. (5) can be solved numerically applying only a very small step $\Delta z$ for the total $z$ region even if the parameters of the layer remain unvariable inside the layers. It is more convenient to consider the model of the crystal as a set of layers having constant parameters inside of each layer. The values of the parameters are changed only at the boundaries between the layers. Equation (5) for the layer with the constant parameters $y_{\varphi}$ has an analytical solution. Such a solution is found in [6] for the Bragg case and in [19] for the Laue case. Here we present the general solution which is valid for both the Bragg case and the Laue case. We assume that the boundary condition does not contain the vanished amplitudes, i.e. $R(0)$ in the Laue case and $R(d)$ in the Bragg case are finite and known. Here $d$ is now the layer thickness. The solution can be written as

$$
R(z)=\frac{F_{n}(z)}{F_{d}(z)}
$$

where

$$
\begin{aligned}
& F_{n}(z)=x_{1}-x_{2} B \exp (-i s \sigma z), \quad F_{d}(z)=1-B \exp (-i s \sigma z), \\
& x_{1,2}=-\frac{s}{C_{1}}\left[-a \pm \sqrt{a^{2}-s C_{1}^{2}}\right], \quad \sigma=\frac{2}{L_{\mathrm{ex}}} \sqrt{a^{2}-s C_{1}^{2}}, \\
& a=y-y_{\varphi}+i y_{0}, \quad B=\frac{\left(x_{1}-R\left(z_{\mathrm{b}}\right)\right)}{\left(x_{2}-R\left(z_{\mathrm{b}}\right)\right)} \exp \left(i \sigma z_{\mathrm{b}}\right) .
\end{aligned}
$$

Hereafter it is assumed that the square root has the positive imaginary part. One can verify the solution by the direct substitution. It is easy to verify the boundary condi- 
tions as well. Equations (10) and (11) allow to derive the recurrent relation for the reflection amplitude at the exit surface $z=z_{\mathrm{e}}$ from the known value at the entrance surface $z=z_{\mathrm{b}}$.

$$
R\left(z_{\mathrm{e}}\right)=\frac{\left(x_{1}-x_{2}\right) R\left(z_{\mathrm{b}}\right)+x_{2}\left[x_{1}-R\left(z_{\mathrm{b}}\right)\right][\exp (i \sigma d)-1]}{x_{1}-x_{2}+\left[x_{1}-R\left(z_{\mathrm{b}}\right)\right][\exp (i \sigma d)-1]} .
$$

Here $z_{\mathrm{e}}=d$ and $z_{\mathrm{b}}=0$ in the Laue case and $z_{\mathrm{e}}=0$ and $z_{\mathrm{b}}=d$ in the Bragg case.

Let us consider the particular cases of kinematical diffraction. As $d \rightarrow 0$, we obtain the approximate relation

$$
R\left(z_{\mathrm{e}}\right)=R\left(z_{\mathrm{b}}\right)+d\left[\frac{2 i a}{L_{\mathrm{ex}}} R\left(z_{\mathrm{b}}\right)-\frac{i C_{1}}{L_{\mathrm{ex}}}\left[1+s R^{2}\left(z_{\mathrm{b}}\right)\right]\right]
$$

We note that Eq. (13) can be obtained directly from Eq. (5) if the right-hand side of the equation at the boundary is taken and a derivative is replaced by $\left[R\left(z_{\mathrm{e}}\right)-R\left(z_{\mathrm{b}}\right)\right] /(-s d)$. In the pure kinematical case when $|R(z)| \ll 1$ and $|a R(z)| \ll 1$ we have a simple expression $R\left(z_{\mathrm{e}}\right)=R\left(z_{\mathrm{b}}\right)-i d C_{1} / L_{\mathrm{ex}}$. This means that the reflection amplitude increases linearly with the thickness of the crystal and the value of the parameter of deviation from the Bragg condition is not important.

Another case of kinematical diffraction may be obtained when the parameter of the deviation from the Bragg condition is large so that $|a| \gg\left|C_{1}\right|$. Under this condition in the Bragg case we have approximately $\sqrt{a^{2}-s C_{1}^{2}} \approx a$. Then $x_{1} \approx 0, x_{2} \approx 2 a / C_{1}$, $\left|x_{2}\right| \gg 1, \sigma d=\Phi=2 a d / L_{\mathrm{ex}}$, and we obtain from Eq. (12) that $R\left(z_{\mathrm{e}}\right)=R\left(z_{\mathrm{b}}\right) \exp (i \Phi)$. This means that the layer changes the phase of the reflection amplitude which may have the large modulus due to reflection at the substrate. The layer does not influence practically the modulus of the reflection amplitude. Concerning the phase, the latter can be measured by means of X-ray standing wave technique (see below).

\subsection{The local transmission amplitude}

Taking into account definition (4), we write the first Takagi equation as follows

$$
\frac{\mathrm{d} E_{0}}{\mathrm{~d} z}=i\left[\frac{\pi \chi_{0}}{\lambda \gamma_{0}}-\frac{C_{1}}{L_{\mathrm{ex}}} R(z)\right] E_{0}(z)
$$

If the function $R(z)$ is known, the solution may be written directly

$$
E_{0}(z)=\exp \left\{i \frac{\pi \chi_{0}}{\lambda \gamma_{0}} z-i \frac{C_{1}}{L_{\mathrm{ex}}} \int_{0}^{z} \mathrm{~d} z^{\prime} R\left(z^{\prime}\right)\right\} E_{0}(0)=T(z) E_{0}(0) .
$$

This solution may be used for numerical calculation, however, again with a disadvantage owing to the integral. If the layer has a constant parameter $y_{\varphi}$, the function $R(z)$ has the analytical solution (10), (11). In this case the integral can be calculated in the analytical form by means of a table integral

$$
\int \frac{\mathrm{d} x}{1-A \exp (\alpha x)}=x-\frac{1}{\alpha} \ln (1-A \exp (\alpha x)) .
$$


As a result, we obtain

$$
T(z)=\exp \left(\frac{i}{2} G z\right) \frac{F_{d}(z)}{F_{d}(0)}
$$

where

$$
G=G^{\prime}+i M=2 \frac{\pi \chi_{0}}{\lambda \gamma_{0}}-2 \frac{C_{1}}{L_{\mathrm{ex}}} x_{1}, \quad M=\frac{\mu_{0}(1-s \beta)}{2 \gamma_{0}}+s \sigma^{\prime \prime} .
$$

Here $\sigma=\sigma^{\prime}+i \sigma^{\prime \prime}, M=G^{\prime \prime}$ and the values $x_{1}$ and $F_{d}(z)$ are defined by Eq. (11). The recurrent relation for the intensity of the transmitted wave is simple. Each layer of thickness $d$ contributes the following factor to the intensity

$$
|T(d)|^{2}=\exp (-M d) \frac{\left|F_{d}(d)\right|^{2}}{\left|F_{d}(0)\right|^{2}} .
$$

To use this expression, one needs to know the value $R\left(z_{\mathrm{b}}\right)$ for this layer. Therefore, this recurrent relation may be used only after the recurrent relation (12) is applied.

\section{The Secondary Radiation Yield}

The X-ray diffractometric technique involves measuring either angular or energy dependence of the reflectivity as a ratio of the intensities of the reflected and incident beams. If the sample contains the crystal-lattice distortions, the reflectivity curve (the rocking curve in the case of angular dependence) has some additional peaks together with the main peak. For the long range distortions of small magnitude, the shape of the main peak may be changed. On the other hand, for the large distortions in a thin subsurface layer one may measure only a small change of rocking curve tails. In this case the standing wave technique is useful and gives an additional structure information just in the region of the main peak. The standing wave technique consists of measuring the intensity of the secondary radiation scattered via incoherent channels (photoelectron emission or fluorescence) under the conditions of X-ray diffraction. Such radiation involves many spherical waves originating from individual atoms of the crystal. If a detector counts all electrons or photons which reach the surface from the depth of the crystal, the probability of the secondary radiation yield must be averaged over the surface. The averaged probability function depends only on the $z$-coordinate, the distance from an atom-source to the surface.

In the case of photons the probability function $P_{\mathrm{yi}}(z)$ is an exponential $P_{\mathrm{yi}}(z)=\exp \left(-\mu_{\mathrm{yi}} z\right)$ where $\mu_{\mathrm{yi}}$ is a linear absorption coefficient of the fluorescent radiation and $z$ is counted from the surface to the volume of the sample. Below we assume that the detector is mounted on the side of the incident beam. When the detector is mounted on the opposite side, we may consider formally a negative value of $\mu_{\mathrm{yi}}$. In the case of electrons the situation is more complicated. However, even in this case the exponential is a rather good approximation to the real function as discussed in [6].

Both the photoelectron emission and the fluorescence are the products of the photoelectron interaction of X-ray wave with an atom. In the dipole approximation the intensity of the secondary radiation yield (SRY) from the atom is proportional to the intensity of the X-ray field at the atom position $[10,11,24]$ and the size of the atom is negligible. To take into account the size of the atom, thermal vibrations, or atom displa- 
cements from an equilibrium position, we have to introduce some small corrections. In the case of fluorescence one can use an energy sensitive detector and separate the signal from the atoms of a definite sort among all the atoms of the crystal. The structure factor of these atoms may be different from the crystal-lattice structure factor.

Let us consider again the layered crystal. The layers are uniform, i.e. they have the constant parameters of distortion. However, these parameters may be different for the various layers. The total yield of secondary radiation $I_{\mathrm{SR}}$ is a sum over all layers

$$
I_{\mathrm{SR}}=\sum_{n=1}^{N} Z_{n-1} I_{\mathrm{SR}}^{(n)}, \quad Z_{n}=\left|E_{0}\left(z_{n}\right)\right|^{2} P_{\mathrm{yi}}\left(z_{n}\right),
$$

where $I_{\mathrm{SR}}^{(n)}$ is a contribution of the layer with the back boundary at $z_{n}\left(z_{0}=0\right)$. The thickness of the $n$-th layer is $d_{n}=z_{n}-z_{n-1}$. The contribution $I_{\mathrm{SR}}^{(n)}$ is given by

$$
\begin{aligned}
I_{\mathrm{SR}}^{(n)}= & \chi_{0 \mathrm{a}}^{\prime \prime} \int_{0}^{d} \mathrm{~d} z^{\prime} P_{\mathrm{yi}}\left(z^{\prime}\right)\left|T\left(z^{\prime}\right)\right|^{2} \\
& \times\left[1+\frac{\left|E_{h}\left(z^{\prime}\right)\right|^{2}}{\left|E_{0}\left(z^{\prime}\right)\right|^{2}}+2 \operatorname{Re}\left\{\frac{E_{h}\left(z^{\prime}\right)}{E_{0}\left(z^{\prime}\right)} C \frac{\chi_{\bar{h}}^{\prime \prime}}{\chi_{0 \mathrm{a}}^{\prime \prime}} \exp \left[i \varphi_{\mathrm{a}}\left(z^{\prime}\right)\right] \exp \left[-W_{\mathrm{a}}\right]\right\}\right],
\end{aligned}
$$

where the index a indicates that the yield is calculated only for the atoms contributing into the secondary radiation yield (SRY). All parameters must be taken for the $n$-th layer. To use the solution (10), we rewrite the expression in terms of the local reflection amplitude, taking into account definition (4)

$$
\begin{aligned}
I_{\mathrm{SR}}^{(n)}= & \chi_{0 \mathrm{a}}^{\prime \prime} \int_{0}^{d} \mathrm{~d} z^{\prime} P_{\mathrm{yi}}\left(z^{\prime}\right)\left|T\left(z^{\prime}\right)\right|^{2} \\
& \times\left[1+\left|R\left(z^{\prime}\right)\right|^{2}|Y|^{2} \beta+2 \operatorname{Re}\left\{R\left(z^{\prime}\right) Y \beta^{1 / 2} C \frac{\chi_{\bar{h} a}^{\prime \prime}}{\chi_{0 \mathrm{a}}^{\prime \prime}} \exp \left[-i \varphi\left(z^{\prime}\right)+i \varphi_{\mathrm{a}}\left(z^{\prime}\right)\right] \exp \left[-W_{\mathrm{a}}\right]\right\}\right] .
\end{aligned}
$$

We accept a reasonable assumption that the difference $\varphi(z)-\varphi_{\mathrm{a}}(z)=\Delta \varphi_{\mathrm{a}}$ does not depend on $z$. If the atom is a source of the SRY and is located at the crystal lattice node, $\Delta \varphi_{\mathrm{a}}=0$. However, in the case of an impurity atom this parameter may be different from zero.

We calculate the integral in the analytical form. First of all, we use the solution (11) for $R(z)$ and write

$$
\begin{aligned}
I_{\mathrm{SR}}^{(n)}= & \frac{\chi_{0 \mathrm{a}}^{\prime \prime}}{\left|F_{d}(0)\right|^{2}} \int_{0}^{d} \mathrm{~d} z \exp \left(-\left[M+\mu_{\mathrm{yi}}\right] z\right) \\
& \times\left[\left|F_{d}(z)\right|^{2}+C_{\mathrm{r}}\left|F_{n}(z)\right|^{2}+\operatorname{Re}\left(C_{\mathrm{i}} F_{d}^{*}(z)\right) F_{n}(z)\right],
\end{aligned}
$$

where

$$
\begin{aligned}
& C_{\mathrm{r}}=|Y|^{2} \beta, \quad C_{\mathrm{i}}=2 C Y \beta^{1 / 2} f_{\mathrm{c}} \exp \left(i \varphi_{\mathrm{c}}\right), \\
& f_{\mathrm{c}}=\frac{\left|\chi_{\bar{h}}^{\prime \prime}\right|}{\chi_{0 \mathrm{a}}^{\prime \prime}} \exp \left(-W_{\mathrm{a}}\right), \quad \varphi_{\mathrm{c}}=\Delta \varphi_{\mathrm{a}}-\arg \left(\chi_{h \mathrm{a}}^{\prime \prime}\right) .
\end{aligned}
$$


The values $f_{\mathrm{c}}$ and $P_{\mathrm{c}}=-\varphi_{\mathrm{c}} / 2 \pi=d_{\mathrm{c}} / d_{\mathrm{hkl}}$ are called the coherent fraction and the relative coherent position of an atom as a source of the SRY [11, 20]. Hereafter $d_{\mathrm{hkl}}$ is a distance between the reflecting atomic planes and $d_{\mathrm{c}}$ is a shift of the atom from the origin of the unit cell along the reciprocal lattice vector. The integrand of Eq. (23) is a sum of the exponentials. Therefore, the integral can be calculated straightforwardly. As a result, we have

$$
I_{\mathrm{SR}}^{(n)}=d \frac{\chi_{0 \mathrm{a}}^{\prime \prime}}{|1-B|^{2}}\left[A_{1} \Psi_{1}+A_{2} \Psi_{2}-\operatorname{Re}\left(A_{3} \Psi_{3}\right)\right],
$$

where $B$ is determined by Eq. (11) and

$$
\begin{aligned}
& \Psi_{k}=\left[1-\exp \left(-a_{k}\right)\right] / a_{k}, \quad k=1,2,3, \\
& a_{1}=\left(M+\mu_{\mathrm{yi}}\right) d, \quad a_{2}=a_{1}-2 s \sigma^{\prime \prime} d, \quad a_{3}=a_{1}+i s \sigma d, \\
& A_{1}=1+\left|x_{1}\right|^{2} C_{\mathrm{r}}+\operatorname{Re}\left(C_{\mathrm{i}} x_{1}\right), \\
& A_{2}=|B|^{2}\left[1+\left|x_{2}\right|^{2} C_{\mathrm{r}}+\operatorname{Re}\left(C_{\mathrm{i}} x_{2}\right)\right], \\
& A_{3}=B\left(2\left[1+x_{1}^{*} x_{2} C_{\mathrm{r}}\right]+\left(C_{\mathrm{i}} x_{1}\right)^{*}+C_{\mathrm{i}} x_{2}\right) .
\end{aligned}
$$

We note that Eq. (25) is valid for both the Bragg case and the Laue case of diffraction. The difference between two cases is only in the sign of symbol $s$.

\section{The Method of the Computer Simulation}

The solution described above is used for elaborating the computer program DSWLC as the MS Windows application for PC. The Fortran code of the program may be used in other operating systems. Below we describe the main features of the program. We assume that the crystal contains $N$ layers. The layers can be different by sorts of atoms, crystal structure and so on. In particular, the extinction length can be different in the various layers due to the different values of $X^{\prime}$. This leads to the different scaling coefficients in Eqs. (7), (8). Since this is inconvenient, we introduce the same value of extinction length $L_{\mathrm{ex}}^{(0)}=\lambda \gamma_{0}\left(\pi \beta^{1 / 2} X_{0}^{\prime}\right)^{-1}$ in all layers where $X_{0}^{\prime}$ is a mean value of $X^{\prime}$. Then we replace the static Debye-Waller factor with the parameter $f_{\mathrm{sc}}=\exp (-W)\left(X^{\prime} / X_{0}^{\prime}\right)$ called the power of scattering by the layer. Such a replacement does not change the Takagi equations.

One may distinguish 11 parameters which characterize the layer completely, namely: 1. $d$ as the thickness of the layer; 2. $\Delta \theta_{\mathrm{B}}$ as the shift of the Bragg angle or $\Delta \hbar \omega_{\mathrm{B}}$ as the shift of the X-ray Bragg energy due to a distortion; 3. $f_{\mathrm{sc}}$ as the power of scattering; 4. $\mu_{0}$ as a linear absorption coefficient of X-rays; $5 . \mu_{\mathrm{yi}}$ as an absorption coefficient of secondary radiation, 6. $p=-X^{\prime \prime} / X^{\prime} ; 7 .|Y| ; 8 . \arg (Y)$; 9. $f_{\mathrm{c}}$ as a coherent fraction; 10. $\varphi_{\mathrm{c}}$ as a phase corresponding to a relative coherent position; 11. $\chi_{0 \mathrm{a}}^{\prime \prime}$ as a power of the SRY.

When the parameter $Y$ has different values in the neighboring layers, the value $Y R$ must be the same at both sides of the boundary between these layers. Therefore, $R_{n}=R_{n \pm 1} Y_{n \pm 1} / Y_{n}$.

In the Bragg case the local reflection amplitude vanishes at the back side of the crystal sample, i.e. $R(t)=0$ where $t=z_{N}$ is a thickness of the sample. The measurable value is the reflectivity $P_{\mathrm{R}}$ defined by $P_{\mathrm{R}}=|Y R(0)|^{2}$. Therefore, at first we have to use 
the recurrent relation (12) $N$ times from the back side to the face side of the crystal. Only afterwards we can calculate the secondary radiation yield $I_{\mathrm{SR}}$ by means of summation in Eq. (20), taking into account Eq. (25) and the recurrent relation for coefficients $Z_{n}$ as

$$
Z_{0}=1, \quad Z_{n+1}=Z_{n}\left|T_{n}\left(d_{n}\right)\right|^{2} \exp \left(-\mu_{\mathrm{yi}}^{(n)} d_{n}\right)
$$

The transmissivity $P_{\mathrm{T}}$ can be calculated using the same value as

$$
P_{\mathrm{T}}=Z_{N} \exp \left(\sum_{n=1}^{N} \mu_{\mathrm{yi}}^{(n)} d_{n}\right) .
$$

In the Laue case $R(0)=0$ and we should use the recurrent relation from the face side to the back side. In this case the reflectivity is not determined completely by the local reflection amplitude due to absorption of the radiation in the crystal. We have $P_{\mathrm{R}}=|Y R(t)|^{2} P_{\mathrm{T}}$. The case when the secondary radiation escapes the crystal from the back side can be calculated within the same method using the negative value of $\mu_{\mathrm{yi}}$. The normalization of the curve to unity background can be made numerically.

So far we have assumed that the incident wave is well collimated and monochromatized. In experiments two different techniques are used. The first technique consists of measuring the angular dependence with the aid of a crystal collimator in the $(n,-n)$ nondispersive scheme (the Bragg case). In this case, using a rather monochromatic characteristic radiation of X-ray tube generators, one can neglect the energy dependence. The angular convolution is only necessary with the reflectivity function $P_{\mathrm{R}}^{(m)}(y)$ of the monochromator in the $y$-scale. If the parameter $X^{\prime}$ of the monochromator coincides with the parameter $X_{0}^{\prime}$ of a sample and $\beta_{m}$ is the parameter of asymmetry of the monochromator, we have the well-known formula (see e.g. [11])

$$
\begin{aligned}
& I_{\mathrm{c}}(y)=\frac{1}{S} \sum_{j} \int \mathrm{d} y_{1} P_{\mathrm{R}}^{(m)}\left(j, y_{1}\right) I\left(j, y+y_{1}\left[\beta_{m} \beta\right]^{1 / 2}\right), \\
& S=\sum_{j} \int \mathrm{d} y_{1} P_{\mathrm{R}}^{(m)}\left(j, y_{1}\right) .
\end{aligned}
$$

Here $j$ is an index of polarization and two polarization states $(\pi, \sigma)$ are assumed to be equivalent (nonpolarized radiation). The notation $I(j, y)$ is used for any of the functions $P_{\mathrm{R}}(j, y), P_{\mathrm{T}}(j, y), I_{\mathrm{SR}}(j, y)$.

To save computing time in the calculation of the convolution (29), it is convenient to calculate the tables of data with a constant step of argument $\Delta y_{P}$ for $P_{\mathrm{R}}^{(m)}(y)$ and $\Delta y_{I}$ for $I(y)$ independently and then to recalculate quickly the table of $I(y)$ by means of spline interpolation to the table with the step $\Delta y_{I}=\Delta y_{P}\left(\beta_{m} \beta\right)^{1 / 2}$. Then the integral for all values $y$ can be calculated by a simple summation.

The second technique is usual in the case of a synchrotron radiation source when the incident wave is well-polarized and the crystal-monochromator is rotated, the sample being kept at the same position. Therefore, the energy dependence is measured while the angular position is the same. In this case the energy profile of the incident wave is determined mainly by the angular divergence of the synchrotron radiation beam through the Bragg condition of the monochromator. The angular divergence is defined by the transverse spatial distribution of the brightness of the synchrotron radiation 
source. It is a normal practice to describe the latter by the Gaussian (see e.g. [25]). As a result, in the $y$-scale we have

$$
I_{\mathrm{c}}(y)=\left(\sigma_{y} \sqrt{\pi}\right)^{-1} \int \mathrm{d} y_{1} \exp \left(-y_{1}^{2} / \sigma_{y}^{2}\right) I\left(j, y+y_{1}\right),
$$

where $\sigma_{y}$ is a parameter of fitting. Sometimes a convolution with the Gaussian is useful in the first case in order to take into account a possible mosaicity of the crystal sample.

As a rule, many of 11 parameters describing each layer are known definitely from the procedure of preparing a sample. There are few parameters which must be determined by fitting the calculated spectra to the experimental spectra. Such parameters are as follows: thickness of the layer $d$, shift of the Bragg angle $\Delta \theta_{\mathrm{B}}$ or energy $\Delta \hbar \omega_{\mathrm{B}}$, and the static Debye-Waller factor $\exp (-W), \exp \left(-W_{\mathrm{a}}\right)=\exp (-W)$ being put in many cases.

The method of fitting used in the program is rather simple. One should minimize the function

$$
\chi^{2}=\sum_{i}\left[I_{\mathrm{c}}\left(\theta_{i}\right)-K I_{\mathrm{ex}}\left(\theta_{i}\right)\right]^{2},
$$

where $I_{\mathrm{ex}}\left(\theta_{i}\right)$ is the table value of the concerned function measured in the experiment and $I_{\mathrm{c}}\left(\theta_{i}\right)$ is the theoretical value calculated for the same function at the same argument. If the normalization of the experimental data is accurate, Eq. (31) should be used with $K=1$. Sometimes the intensity of the incident beam is not measured and the experimental data (the X-ray reflectivity or the secondary radiation yield) are known without an accurate normalization. In these cases the program calculates the normalization constant $K$ from the condition of the minimum $\chi^{2}$ value as a function of $K$. The solution is $K=\left(\sum_{i} I_{\mathrm{ex}}^{2}\left(\theta_{i}\right)\right)^{-1} \sum_{i} I_{\mathrm{c}}\left(\theta_{i}\right) I_{\mathrm{ex}}\left(\theta_{i}\right)$.

\section{Epitaxial Film of $\operatorname{In}_{0.5} \mathrm{Ga}_{0.5} P$ on a GaAs Substrate}

As a first example of application of the program DSWLC, we consider the system consisting of a relatively thick epitaxial film of $\operatorname{In}_{0.5} \mathrm{Ga}_{0.5} \mathrm{P}$ on a substrate of GaAs. The results of the experimental study of this system together with the previous theoretical analysis are reported in Ref. [22]. The experiment was done in the two-crystal nondispersive scheme $(n,-n)$, with the (111) reflection of the $\mathrm{Cu} \mathrm{K} \mathrm{K}_{\alpha}$ radiation. The Ge crystal having the asymmetry factor $\beta_{m}=0.04$ is employed as a monochromator. The X-ray reflectivity is measured within the wide range of the angular parameter. The secondary fluorescent radiation $\mathrm{In} \mathrm{L}_{\alpha}$ and $\mathrm{P} \mathrm{K}_{\alpha}$ is measured inside the region of the reflectivity maximum of the film. The experimental data are normalized accurately and shown in Figs. 1 and 2 as circles. The data are taken from Ref. [22]. No information about the diffuse scattering is known. However, one may assume that the diffuse scattering influences the shape of the curves only on the tails of both the main and additional peaks of the reflectivity where the diffraction scattering is small.

We calculate almost all parameters of the epitaxial structure necessary for the computer simulation using the data from the literature. The exceptions are the depth profiles of the crystal lattice spacing $\Delta d_{\mathrm{hkl}} / d_{\mathrm{hkl}}=-\Delta \theta_{\mathrm{B}} / \tan \theta_{\mathrm{B}}=-4.13 \Delta \theta_{\mathrm{B}}$ and the static Debye-Waller factor $\exp (-W)$. We note that the coherent fraction $f_{c}$ is taken as a product of the thermal Debye-Waller factor and the static Debye-Waller factor. The In atoms 


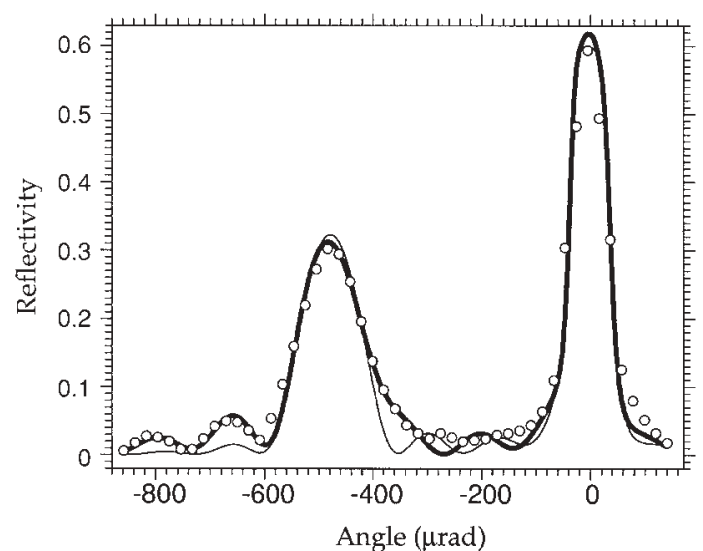

Fig. 1. Angular dependence of the X-ray reflectivity for the epitaxial structure $\mathrm{In}_{0.5} \mathrm{Ga}_{0.5} \mathrm{P} / \mathrm{GaAs}$. The calculation is made in the two-layer approximation (thin line) and 3-layer approximation (thick line). See the parameters in Table 1 . The experimental data are shown by the circles

and the $\mathrm{P}$ atoms have the same coherent fraction. According to our approach we must find the parameters of the multilayered crystal structure. The simplest model contains two layers. One of them describes the epitaxial film and the other describes the substrate. We have found the necessary parameters from fitting only the reflectivity curve of the Fig. 1. The values of the parameters $d$ in $\mu \mathrm{m}, \Delta \theta_{\mathrm{B}}$ in $\mu \mathrm{rad}$, and $\exp (-W)$ are shown in Table 1 together with the value of the fitting parameter $\chi^{2}$. The reflectivity curve calculated for this model is shown in Fig. 1 by the thin line. The calculated curves of the In $\mathrm{L}_{\alpha}$ and $\mathrm{P} \mathrm{K}_{\alpha}$ fluorescence for the same parameters as the reflectivity are shown in Fig. 2 (thin line). One may see that the coincidence between the experimental data and the theoretical curves is rather good. However, difference is apparent. Such a model was used in Ref. [22] where the same result is obtained for the reflectivity. We note that the $\mathrm{P} \mathrm{K}_{\alpha}$ fluorescence yield is calculated with the value of $\varphi_{\mathrm{c}}=-\pi / 2$ $\left(P_{\mathrm{c}}=1 / 4\right)$. Therefore, the polarity of the crystal is such that the In atoms lie on the surface.

As was pointed out in Ref. [22], the two-layer model cannot describe the specific features of the X-ray reflectivity on the left-hand side of the spectrum where significant additional peaks are measured. In this work we try to describe these features using three-layer model. Again the parameters are found from fitting only the X-ray reflectivity curve. Their values are shown in Table 1 . The result of calculating X-ray reflectivity for this model is shown by the thick line in Fig. 1. One may see that the coincidence of

Table 1

The parameters of multilayered structure $\mathrm{In}_{0.5} \mathrm{Ga}_{0.5} \mathrm{P} / \mathrm{GaAs}$

\begin{tabular}{llcl}
\hline$d(\mu \mathrm{m})$ & $\Delta \theta_{\mathrm{B}}(\mu \mathrm{rad})$ & $\exp (-W)$ & fit \\
\hline \multicolumn{3}{c}{ two-layer approximation } \\
0.63 & -480 & 0.84 & 0.056 \\
300 & 0 & 1 & \\
& & three-layer approximation & \\
0.5 & -450 & 0.92 & 0.037 \\
0.13 & -728 & 0.89 & \\
300 & 0 & 1 & \\
\hline
\end{tabular}




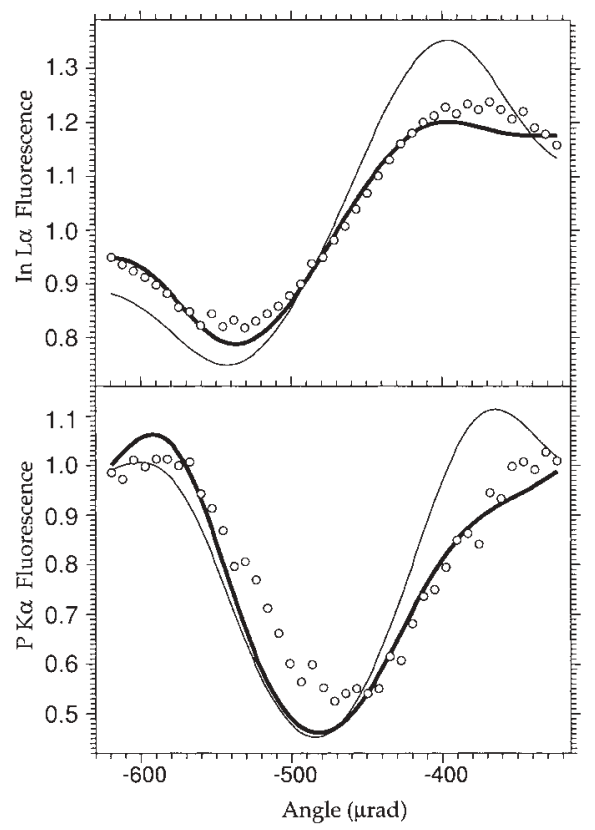

Fig. 2. Angular dependence of In $\mathrm{L}_{\alpha}$ (top picture) and $\mathrm{PK}_{\alpha}$ (bottom picture). The calculation is made for the same parameters as in Fig. 1

the calculated curve with the experimental data becomes much better. Only some small difference exists on the left slope of the epitaxial film peak as well as inside the region between two peaks. The agreement for the In $\mathrm{L}_{\alpha}$ fluorescence yield becomes much better as well. As for the $\mathrm{P} \mathrm{K}_{\alpha}$ fluorescence yield, the only right-hand side of the curve becomes better fitted whereas the left-hand side remains significantly distinctive. Unfortunately, it is difficult to explain this result. At least, it means that the fluorescent data are more sensitive to small differences in the deformation and disorder profiles.

Of course, the four-layer model, five-layer model and so on can be analyzed. We believe that the quality of fitting can be improved in such a way. However, a fine analysis is not the aim of this work. Here we want to discuss a physical reason of the result obtained within the three-layer model. We have found that the epitaxial film is not homogeneous. The region beside the surface has the smaller difference of the lattice spacing $\Delta d_{\mathrm{hkl}} / d_{\mathrm{hkl}}=1.9 \cdot 10^{-3}$ whereas the region beside the substrate has the larger difference $\Delta d_{\mathrm{hkl}} / d_{\mathrm{hkl}}=3.0 \cdot 10^{-3}$. Such a structure can arise due to strong deformation of the film near the substrate. Of course, the lattice spacing is determined by the elastic constants. Very simple qualitative arguments look as follows. Because the substrate has a smaller unit cell volume, the unit cell of the film near the substrate is transformed from the cube to the rectangular parallelepiped. The spacing along the interface inside the layer becomes the same as the spacing inside the substrate. However, the volume of the film unit cell is greater than the substrate one. Therefore, the spacing in the direction normal to the interface (along the reciprocal lattice vector) must be larger. It is this spacing that influences the X-ray diffraction data.

\section{Complete Switching the Beam from the Incident Direction to the Direction of Reflection}

In this section we want to demonstrate an application of the program to many layers. The model of a layered crystal with many layers allows a simulation of macroscopic deformation of the sample. To demonstrate this possibility, we consider the symmetrical Laue case of X-ray diffraction in a silicon crystal where the Bragg angle increases (decreases) linearly with increasing a depth of location of the atomic planes from the entrance to the exit surfaces. Such deformation corresponds approximately to an external distortion like a constant temperature gradient or an excitation of ultrasound vibra- 


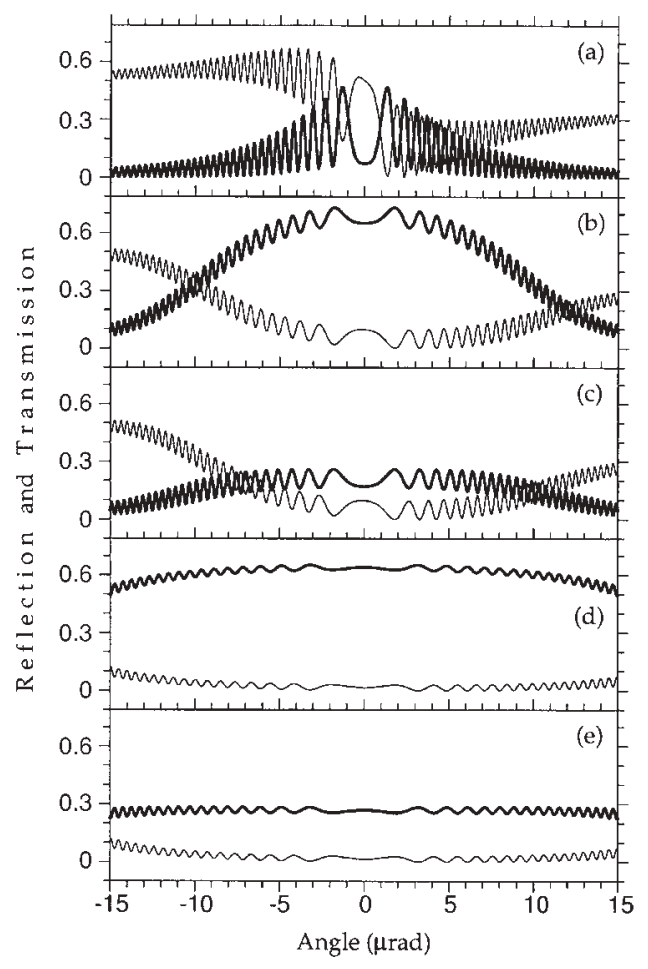

Fig. 3. Angular dependence of the reflectivity (thick line) and transmissivity (thin line) in the symmetrical Laue case of the (220) X-ray diffraction of the Mo $\mathrm{K}_{\alpha}$ radiation in the Si crystal of thickness $584 \mu \mathrm{m}$. a) Undistorted crystal, b) $\Delta \theta_{B}$ changes linearly with the depth from 10 to $-10 \mu \mathrm{rad}, \mathrm{c})$ from -10 to $10 \mu \mathrm{rad}$, d) from 20 to $-20 \mu \mathrm{rad}$, e) from -20 to $20 \mu \mathrm{rad}$

tions by the wave having a half of the period per sample thickness. We note that the Laue case of diffraction in the crystal having a weak gradient of a lattice parameter was investigated by Hildebrandt [26] both experimentally and theoretically within the two-layer model.

Figure $3 \mathrm{a}$ shows the angular dependence of the reflectivity (thick line) and the transmissivity (thin line) in the symmetrical Laue case of (220) diffraction of Mo $K_{\alpha}$ radiation in the perfect (undistorted) crystal plate of thickness $584 \mu \mathrm{m}$. It is assumed that the incident plane wave is $\sigma$-polarized. This allows one to consider more simple and regular picture. As is known, two Bloch waves are excited in the crystal in the Laue case of diffraction. They correspond to the two different branches of dispersion surface and have different speeds of light. Such a difference depends on the parameter of the deviation from the Bragg condition. As a result, the phase difference between the Bloch waves depends on the crystal plate thickness $t$ as well as on the crystal angular position $\Delta \theta$. As for the reflectivity and transmissivity curves, they oscillate with a variable period as a function of $\Delta \theta$. The period depends on the crystal thickness. It decreases with increasing thickness. The crystal is thick so that $\mu_{0} t / \gamma_{0}=0.84$ where $\mu_{0}$ is a linear absorption coefficient and $\gamma_{0}=\cos \theta_{\mathrm{B}}$. The real absorption and the degree of excitation of different Bloch waves depend on the angular deviation. In addition, the absorption of one Bloch wave becomes much smaller at the accurate Bragg position (zero point in Fig. 3). This phenomenon is known as the Borrmann effect [27]. The absorption of the other Bloch wave becomes twice larger. This is due to absorption so that the transmissivity does not exceed $60 \%$ even in the case when the Bragg condition is not fulfilled.

Due to the structure of the Bloch waves the reflectivity has the maximum values when the transmissivity has the minimum values and vice versa. This fact is easy to understand from the energy conservation. Both Bloch waves contribute the same value into the intensity of the reflected beam. Therefore, the reflectivity vanishes at the angular points of destructive interference. However, the intensity contributions of the different Bloch waves are the product of two terms, namely, the reflection component and the degree of excitation. These components are different for the different Bloch waves 
on the tails of the angular dependence. One Bloch wave has the smaller reflection component but the larger degree of excitation. The other Bloch wave has the larger reflection component, but it is excited with the smaller degree. Unfortunately, this cannot be seen directly in the formulae presented above. However, a detailed analysis of the formulae is simple and can be made by the reader.

Figure $3 b$ shows the angular dependence of the reflectivity and transmissivity for the same sample but distorted so that the shift of the Bragg angle is determined by the function $\Delta \theta_{\mathrm{B}}(z)=(10-20 z / t) \mu \mathrm{rad}$. In reality, this function $\Delta \theta_{\mathrm{B}}(z)$ was simulated by the model of a crystal containing 100 layers of the same structure with $\Delta \theta_{\mathrm{B}}$ constant within the layer, but changing by $-0.2 \mu \mathrm{rad}$ at each boundary between two layers. It is easy to see a drastic change of the reflectivity and transmissivity. First of all, the amplitude of oscillations becomes approximately constant within the angular range considered. Its value corresponds to the angular points $\pm 10 \mu \mathrm{rad}$ in Fig. 3a. In the central part of the plot the transmissivity is close to zero and the reflectivity reaches a maximum possible value involving the X-ray absorption in the crystal. Hence we have simulated the phenomenon of the complete switching of the X-ray beam from the incident direction to the direction of reflection. Such an effect was calculated for the first time in Ref. [28] by means of numerical solution of the Takagi equations and independently was observed experimentally for the first time in Ref. [29].

The detailed analytical analysis of this effect can be made within the approximation of the geometrical optics developed by Penning and Polder (see e.g. [30]). However, it is not the aim of this article. We discuss the phenomena taking place in the central angular position of the crystal. At the entrance surface where $\Delta \theta_{\mathrm{B}}=10 \mu \mathrm{rad}$ the weakly absorbing Bloch wave is well-excited but has a small reflection component. However, the shift of the Bragg angle $\Delta \theta_{\mathrm{B}}$ becomes smaller and smaller with increasing the depth $z$ and then it changes a sign. This leads to increasing the reflection component of the Bloch wave. The degree of excitation remains unchanged. The point is that the interaction with the other Bloch wave is small as the deformation varies slowly. As a result, at the exit surface this Bloch wave becomes well excited and has the large reflection component. The excitation of the other Bloch wave is small at the entrance surface and the reflection component becomes small at the exit surface. That is why the amplitude of oscillations is small too. The effect of decreasing the transmissivity follows from the energy conservation. Thus, only one Bloch wave is well-excited for all depth $z$ inside the crystal and this wave is weakly absorbing.

Let us consider the situation when the Bragg angle changes according to the formula $\Delta \theta_{\mathrm{B}}(z)=(-10+20 z / t) \mu \mathrm{rad}$. It is easy to understand that the other Bloch wave is well-excited and strongly absorbing. Therefore, the complete switching of the beam takes place too, but the reflectivity is smaller than in the first case due to strong absorption. The amplitude of oscillations is slightly larger than in Fig. $3 \mathrm{~b}$ because the weakly excited wave is weakly absorbing. Therefore, its amplitude is larger at the exit surface. As the gradient of distortion increases, the phenomenon becomes more pronounced. Such a conclusion is verified in Figs. $3 \mathrm{~d}$ and e where $\Delta \theta_{\mathrm{B}}$ varies from 20 to $-20 \mu \mathrm{rad}$ and from -20 to $20 \mu \mathrm{rad}$, correspondingly. We note that the effect of complete switching the incident beam can be obtained within a rather large angular region which is much larger than the angular region of the X-ray dynamical diffraction in a perfect (undistorted) crystal. 


\section{Conclusion}

The method of the computer simulation of the angular dependence of the reflectivity, transmissivity and yield of secondary radiation under the condition of the dynamical Xray diffraction is presented for the multilayered crystal systems or crystals with a onedimensional profile of the crystal-lattice distortion. The method is based on an analytical solution of the diffraction problem inside the individual layer. A set of the parameters describing completely each layer is formulated. The formulae are derived in a form applicable for both the Bragg case and the Laue case of X-ray diffraction.

The computer program DSWLC is elaborated as a Microsoft Windows application. The program was applied to the numerical analysis of two problems among many others. In the first problem the structure parameters of the epitaxial film $\operatorname{In}_{0.5} \mathrm{Ga}_{0.5} \mathrm{P}$ on the substrate of GaAs are found within the larger accuracy than in the previous publication. It is shown that the model with a nonuniform lattice spacing along the depth of the film gives the better coincidence of the theoretical curves with the experimental data. In the second problem it is shown that the model of the multilayered crystal allows to simulate the phenomenon of the complete switching of the beam from the incident direction to the direction of reflection. It is shown that the angular region of the complete switching becomes much larger for the large distortion compared to the perfect crystal.

Acknowledgement The work is supported by the Russian Foundation for Basic Research (project No. 01-02-16508).

\section{References}

[1] B. W. Batterman, Appl. Phys. Lett. 1, 68 (1962); Phys. Rev. A 133, 759 (1964).

[2] J. A. Golovchenko, B. W. Batterman, and W. L. Brown, Phys. Rev. B 10, 4239 (1974).

[3] V. N. Shchemelev, M. V. Kruglov, and V. P. Pronin, Fiz. Tverd. Tela 12, 2495 (1970); Sov. Phys. - Solid State 12, 2005 (1971).

[4] V. N. ShChemelev and M. V. Kruglov, Fiz. Tverd. Tela, 14, 3556 (1972); Sov. Phys. - Solid State 14, 2988 (1973).

[5] M. V. Kovalchuk, D. Liljequist, and V. G. Kohn, Fiz. Tverd. Tela 28, 3409 (1986); Sov. Phys. - Solid State 28, 1918 (1986).

[6] M. V. Kovalchuk, V. G. Kohn, and E. F. Lobanovich, Fiz. Tverd. Tela 27, 3379 (1985); Sov. Phys. - Solid State 27, 2034 (1985).

[7] S. K. Andersen, J. A. Golovchenko, and G. Mair, Phys. Rev. Lett. 37, 1141 (1976).

[8] M. V. Kruglov, V. N. Shchemelev, and G. G. Kareva, phys. stat. sol. (a) 46, 343 (1978).

[9] E. A. Sozontov, M. V. Kruglov, and B. G. ZaKharov, phys. stat. sol. (a) 66, 303 (1981).

[10] A. M. Afanasev and V. G. Kohn, Zh. Eksp. Teor. Fiz. 74, 300 (1978); Sov. Phys. - JETP 47, 154 (1978).

[11] M. V. KovalchuK and V. G. Kohn, Usp. Fiz. Nauk 149, 69 (1986); Sov. Phys. - Usp. 29, 426 (1986).

[12] J. Zegenhagen, Surf. Sci. Rep. 18, 199 (1993).

[13] A. Yu. Kazimirov, M. V. Kovalchuk, A. N. Sosphenov, V. G. Kohn, J. Kub, P. Novak, M. Nevriva, and J. CermaK, Acta Cryst. B 48, 577 (1992).

[14] A. Lessmann, S. Brennan, B. Materlik, M. Schuster, H. Riechert, Rev. Sci. Instrum. 66, 1428 (1995).

[15] A. Kazimirov, T. Haage, L. Ortega, A. Stierle, F. Comin, and J. Zegenhagen, Solid State Commun. 104, 347 (1997).

[16] A. Kazimirov, J. Zegenhagen, and M. Cardona, Science 282, 930 (1998). 
[17] E. Sozontov, L. X. Cad, A. Kazimirov, V. Kohn, M. Cardona, and J. Zegenhagen, Phys. Rev. Lett. 86, 5329 (2001).

[18] T. God, A. Hill, D. Bahr, and G. Materlik, Rev. Sci. Instrum. 66, 1522 (1995).

[19] A. Yu. Kazimirov, M. V. Kovalchuk, and V. G. Kohn, Acta Cryst. A 46, 649 (1990).

[20] M. J. BedzyK and G. Materlik, Phys. Rev. B 32, 6456 (1985).

[21] V. G. KoHn and M. V. KovalchuK, phys. stat. sol. (a) 64, 359 (1981).

[22] A. Yu. Kazimirov, M. V. KovalchuK, and V. G. Kohn, Pisma Zh. Tekh. Fiz. 14, 1345 (1988); Sov. Tech. Phys. Lett. 14, 587 (1988).

[23] A. M. Afanasev, M. V. KovalchuK, E. K. Kovev, and V. G. Kohn, phys. stat. sol. (a) 42, 415 (1977).

[24] I. A. Vartanyants and J. Zegenhagen, Solid State Commun. 113, 299 (2000).

[25] V. Kohn, I. Snigireva, and A. Snigirev, Phys. Rev. Lett. 85, 2745 (2000).

[26] G. HildebrandT, Z. Kristallogr. 112, 312 (1959).

[27] G. Borrmann, Z. Phys. 42, 157 (1941); 127, 297 (1950).

[28] E. A. Tikhonova, Metallofiz. 2, 32 (1980); 3, 18 (1981).

[29] A. R. Mkrtchyan, M. A. Navasardyan, and V. K. Mirzoyan, Pisma Zh. Tekh. Fiz. 8, 677 (1982).

[30] Z. G. PinsKer, Dynamical Scattering of X-rays in Crystals, Springer-Verlag, Heidelberg 1978. 